\title{
Design Intelligent Robust Model-base Sliding Guidance Controller for Spherical Motor
}

\author{
Mojtaba Yaghoot, Farzin Piltan, Meysam Esmaeili, Mohammad Ali Tayebi, Mahsa Piltan \\ Research and Development Department, Institute of Advance Science and Technology-IRAN SSP, Shiraz/Iran, \\ http://WWW.IRANSSP.COM \\ Email: SSP.ROBOTIC@IRANSSP.COM
}

\begin{abstract}
Stability, robust and reliability are the main objectives to design a controller for highly nonlinear spherical motor. Most of linear and nonlinear controllers are stable, model-base controllers are reliable but in this group sliding mode controller is a robust controller. Therefore in this research sliding mode controller is used to design stable, robust and reliable controller. For intelligence part, the minimum rule base Proportional Integral Derivative (PID) Fuzzy hybrid guidance Controller for three dimensions spherical motor is presented in this research. Guidance control in a three dimensions spherical motor is performed by the robust sliding mode controllers producing the control signals which are applied to systems torque. Sliding mode controller has an important problem, namely chattering. In this research, chattering-free sliding mode controller is design as a robust guidance controller to their robust performance in a wide range of operating conditions. In this research the PID-like fuzzy controller can be constructed as a parallel structure of a PD-like fuzzy controller and a PI-like controller to have the minimum rule base. Nonlinear type robust sliding mode controller is used to modify PID fuzzy logic theory to design robust and reliable hybrid guidance methodology. This research is used to reduce or eliminate the fuzzy and conventional sliding mode controller problem based on minimum rule base fuzzy logic theory and modified it by sliding mode method to control of spherical motor system.
\end{abstract}

Index Terms - Fuzzy Hybrid guidance control, robust sliding mode controller, PID like fuzzy control, PD like fuzzy control, PI like control, three dimension spherical motor, chattering-free controller.

\section{INTRODUCTION}

Multi-degree-of-freedom (DOF) actuators are finding wide use in a number of Industries. Currently, a significant number of the existing robotic actuators that can realize multi-DOF motion are constructed using gear and linkages to connect several single-DOF motors in series and/or parallel. Not only do such actuators tend to be large in size and mass, but they also have a decreased positioning accuracy due to mechanical deformation, friction and backlash of the gears and linkages. A number of these systems also exhibit singularities in their workspaces, which makes it virtually impossible to obtain uniform, high-speed, and high-precision motion. For high precession trajectory planning and control, it is necessary to replace the actuator system made up of several singleDOF motors connected in series and/or parallel with a single multi-DOF actuator. The need for such systems has motivated years of research in the development of unusual, yet high performance actuators that have the potential to realize multi-DOF motion in a single joint. One such actuator is the spherical motor. Compared to conventional robotic manipulators that offer the same motion capabilities, the spherical motor possesses several advantages. Not only can the motor combine 3-DOF motion in a single joint, it has a large range of motion with no singularities in its workspace. The spherical motor is much simpler and more compact in design than most multiple single-axis robotic manipulators. The motor is also relatively easy to manufacture. The spherical motor have potential contributions to a wide range of applications such as coordinate measuring, object tracking, material handling, automated assembling, welding, and laser cutting. All these applications require high precision motion and fast dynamic response, which the spherical motor is capable of delivering. Previous research efforts on the spherical motor have demonstrated most of these features. These, however, come with a number of challenges. The spherical motor exhibits coupled, nonlinear and very complex dynamics. The design and implementation of feedback controllers for the motor are complicated by these dynamics. The controller design is further complicated by the orientation-varying torque generated by the spherical motor. Some of these challenges have been the focus of previous and ongoing research [1-11].

Controller (control system) is a device which can sense information from linear or nonlinear system (e.g., three degrees of freedom spherical motor) to improve the systems performance and the immune system behavior [11-20]. In feedback control system considering that there are many disturbances and also variable dynamic parameters something that is really necessary is keeping plant variables close to the desired value. Feedback control system development is the most important thing in many different fields of safety engineering. The main targets in design control systems are safety stability, good disturbance rejection to reach the best safety, and small tracking error[21-26]. At present, in some applications spherical motors are used in unknown and unstructured 
environment, therefore strong mathematical tools used in new control methodologies to design nonlinear robust controller with an acceptable safety performance (e.g., minimum error, good trajectory, disturbance rejection). According to the control theory, systems' controls are divided into two main groups: conventional control theory and soft computing control theory. Conventional control theories are work based on system dynamic model. This technique is highly sensitive to the knowledge of all parameters of nonlinear spherical motor's dynamic equation. Conventional control theory is divided into two main groups: linear control theory and nonlinear control theory. Soft computing (intelligent) control theory is free of some challenges associated to conventional control theory. This technique is worked based on intelligent control theory. This theory is divided into the following groups: fuzzy logic theory, neural network theory, genetic algorithm and neuro-fuzzy theory.

A nonlinear methodology is used for nonlinear uncertain systems (e.g., spherical motor) to have an acceptable performance. These controllers divided into six groups, namely, feedback linearization (computedtorque control), passivity-based control, sliding mode control (variable structure control), artificial intelligence control, lyapunov-based control and adaptive control[1326]. Sliding mode controller (SMC) is a powerful nonlinear controller which has been analyzed by many researchers especially in recent years. This theory was first proposed in the early 1950 by Emelyanov and several co-workers and has been extensively developed since then with the invention of high speed control devices [12-18]. The main reason to opt for this controller is its acceptable control performance in wide range and solves two most important challenging topics in control which names, stability and robustness [24-66]. Sliding mode controller is divided into two main sub controllers: discontinues controller $\left(\boldsymbol{\tau}_{\text {dis }}\right)$ and equivalent controller $\left(\boldsymbol{\tau}_{\boldsymbol{e q}}\right)$. Discontinues controller causes an acceptable tracking performance at the expense of very fast switching. In the theory of infinity fast switching can provide a good tracking performance but it also can provide some problems (e.g., system instability and chattering phenomenon). After going toward the sliding surface by discontinues term, equivalent term help to the system dynamics match to the sliding surface[61-66]. However, this controller used in many applications but, pure sliding mode controller has following challenges: chattering phenomenon, and nonlinear equivalent dynamic formulation [20]. Chattering phenomenon can causes some problems such as saturation and heat the mechanical parts of spherical motor. To reduce or eliminate the chattering, various papers have been reported by many researchers which classified into two most important methods: boundary layer saturation method and estimated uncertainties method [22-36]. In boundary layer saturation method, the basic idea is the discontinuous method replacement by saturation (linear) method with small neighborhood of the switching surface. This replacement caused to increase the error performance against with the considerable chattering reduction.

Although the fuzzy-logic control is not a new technique, its application in this current research is considered to be novel since it aimed for an automated dynamic-less response rather than for the traditional objective of uncertainties compensation[22-26]. The intelligent tracking control using the fuzzy-logic technique provides a cost-and-time efficient control implementation due to the automated dynamic-less input. This in turn would further inspire multi-uncertainties testing for continuum robot [55-66]. In project we can use fuzzy logic theory when a plant can be considered as a black box with outputs available for measurement and a possibility of changing inputs. The plant is supposed to be observable and controllable. Some information about the plant operation or plant control is available, which can or cannot be of a quantitative nature, but it can be formulated as a set of rules (maybe after some processing). An acceptable fuzzy control solution is possible, which should satisfy design specifications. It must not be optimal in regard to some criteria as it is hard to prove that a fuzzy control system is optimal and even stable. However, a fuzzy controller is able to provide a stable and 'good' solution.

The methodology of boundary layer saturation method is achieve accurate tracking for non-linear and time varying system in presence of disturbance and parameter variations based on continuous feedback control law. To rectify the chattering phenomenon, the saturation continuous control is introduced. Figure 1 shows the linear saturation boundary layer function.

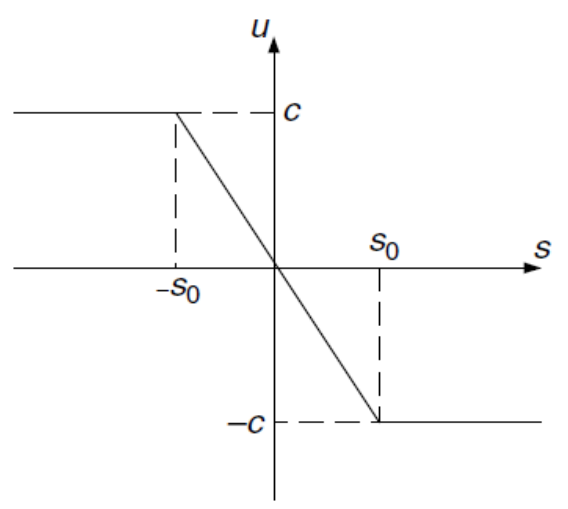

Fig 1: Linear Saturation Boundary Layer Functions

Saturation boundary layer method has some disadvantages such as increase the error and reduces the speed of response. To solve linear boundary layer saturation challenge, design nonlinear intelligent saturation boundary layer function instead of linear saturation boundary method is introduced. This method is used to reduce or eliminate the chattering as well as reduce the error performance. However the design sliding mode fuzzy controller to reduce the chattering is faster and robust than sliding mode controller based on linear boundary layer method but adjust the fuzzy logic input 
and output gain updating factor is very difficult. Figure 2 shows the nonlinear artificial intelligence sliding mode controller based on fuzzy logic methodology.

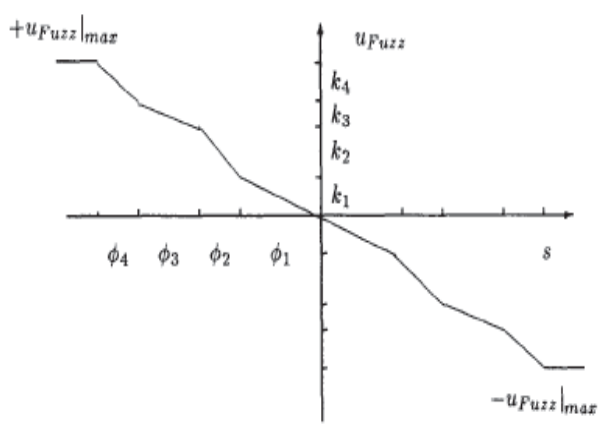

Fig 2: Nonlinear Fuzzy Saturation Boundary Layer Functions

The main idea in this research is focused on design minimum rule base Proportional Integral Derivative (PID) Fuzzy robust sliding mode hybrid guidance Controller for three dimensions spherical motor. This research has two important objectives:

1. Reduce the number of rule base in PID fuzzy controller.

2. Design chattering free sliding mode guidance of fuzzy logic theory based on hybrid theory.

This paper is organized as follows; section 2, is served as an introduction to the dynamic of three degrees of freedom spherical motor, design sliding mode controller with application to spherical motor and design and introducing of fuzzy inference system. Part 3, introduces and describes the methodology algorithm. Section 4 presents the simulation results and discussion of this algorithm applied to three degrees of freedom spherical motor and the conclusion is described as the last section.

\section{THEORY}

Dynamic and Kinematics Formulation of Spherical Motor: Dynamic modeling of spherical motors is used to describe the behavior of spherical motor such as linear or nonlinear dynamic behavior, design of model based controller such as pure sliding mode controller which design this controller is based on nonlinear dynamic equations, and for simulation. The dynamic modeling describes the relationship between motion, velocity, and accelerations to force/torque or current/voltage and also it can be used to describe the particular dynamic effects (e.g., inertia, coriolios, centrifugal, and the other parameters) to behavior of system[1-10]. Spherical motor has nonlinear and uncertain dynamic parameters 3 degrees of freedom (DOF) motor.

The equation of a spherical motor governed by the following equation [1-10]:

$$
H(q)\left[\begin{array}{c}
\ddot{\alpha} \\
\ddot{\beta} \\
\ddot{\gamma}
\end{array}\right]+\mathrm{B}(q)\left[\begin{array}{c}
\dot{\alpha} \dot{\beta} \\
\dot{\alpha} \dot{\gamma} \\
\dot{\beta} \dot{\gamma}
\end{array}\right]+C(q)\left[\begin{array}{c}
\dot{\alpha}^{2} \\
\dot{\beta}^{2} \\
\dot{\gamma}^{2}
\end{array}\right]=\left[\begin{array}{c}
\tau_{x} \\
\tau_{y} \\
\tau_{z}
\end{array}\right]
$$

Where $\tau$ is actuation torque, $H(q)$ is a symmetric and positive define inertia matrix, $\mathrm{B}(\mathrm{q})$ is the matrix of coriolios torques, $\mathrm{C}(\mathrm{q})$ is the matrix of centrifugal torques This is a decoupled system with simple second order linear differential dynamics. In other words, the component $\ddot{q}$ influences, with a double integrator relationship, only the variable $q_{i}$, independently of the motion of the other parts. Therefore, the angular acceleration is found as to be [1-11]:

$$
\ddot{\mathrm{q}}=\mathrm{H}^{-1}(\mathrm{q}) \cdot\{\tau-\{\mathrm{B}+\mathrm{C}\}\}
$$

This technique is very attractive from a control point of view.

Study of spherical motor is classified into two main groups: kinematics and dynamics. Calculate the relationship between rigid bodies and final part without any forces is called Kinematics. Study of this part is pivotal to design with an acceptable performance controller, and in real situations and practical applications As expected the study of kinematics is divided into two main parts: forward and inverse kinematics. Forward kinematics has been used to find the position and orientation of task frame when angles of joints are known. Inverse kinematics has been used to find possible joints variable (angles) when all position and orientation of task frame be active [1].

The main target in forward kinematics is calculating the following function:

$$
\Psi(X, q)=0
$$

Where $\Psi(.) \in R^{n}$ is a nonlinear vector function, $X=\left[X_{1}, X_{2}, \ldots \ldots, X_{l}\right]^{T}$ is the vector of task space variables which generally task frame has three task space variables, three orientation, $q=\left[q_{1}, q_{2}, \ldots, q_{n}\right]^{T}$ is a vector of angles or displacement, and finally $n$ is the number of actuated joints. The Denavit-Hartenberg (D-H) convention is a method of drawing spherical motor free body diagrams. Denvit-Hartenberg (D-H) convention study is necessary to calculate forward kinematics in this motor.

A systematic Forward Kinematics solution is the main target of this part. The first step to compute Forward Kinematics (F.K) is finding the standard D-H parameters. The following steps show the systematic derivation of the standard D-H parameters.

1. Locate the spherical motor

2. Label joints

3. Determine joint rotation $(\theta)$

4. Setup base coordinate frames.

5. Setup joints coordinate frames. 
6. Determine $\alpha_{i}$, that $\alpha_{i}$, link twist, is the angle between $Z_{i}$ and $Z_{i+1}$ about an $X_{i}$.

7. Determine $d_{i}$ and $a_{i}$, that $a_{i}$, link length, is the distance between $Z_{i}$ and $Z_{i+1}$ along $X_{i} . d_{i}$, offset, is the distance between $X_{i-1}$ and $X_{i}$ along $Z_{i}$ axis.

8. Fill up the D-H parameters table. The second step to compute Forward kinematics is finding the rotation matrix $\left(R_{n}^{0}\right)$. The rotation matrix from $\left\{F_{i}\right\}$ to $\left\{F_{i-1}\right\}$ is given by the following equation;

$$
\mathrm{R}_{\mathrm{i}}^{\mathrm{i}-1}=\mathrm{U}_{\mathrm{i}\left(\theta_{\mathrm{i}}\right)} \mathrm{V}_{\mathrm{i}\left(\alpha_{\mathrm{i}}\right)}
$$

Where $U_{i\left(\theta_{i}\right)}$ is given by the following equation [1-11];

$$
U_{i}\left(\theta_{i}\right)=\left[\begin{array}{ccc}
\cos \left(\theta_{\mathrm{i}}\right) & -\sin \left(\theta_{\mathrm{i}}\right) & 0 \\
\sin \left(\theta_{\mathrm{i}}\right) & \cos \left(\theta_{\mathrm{i}}\right) & 0 \\
0 & 0 & 1
\end{array}\right]
$$

and $V_{i\left(\alpha_{i}\right)}$ is given by the following equation [1-11];

$$
V_{i\left(\theta_{i}\right)}=\left[\begin{array}{ccc}
1 & 0 & 0 \\
0 & \cos \left(\alpha_{i}\right) & -\sin \left(\alpha_{i}\right) \\
0 & \sin \left(\alpha_{i}\right) & \cos \left(\alpha_{i}\right)
\end{array}\right]
$$

So $\left(R_{n}^{0}\right)$ is given by [8]

$$
\mathrm{R}_{\mathrm{n}}^{0}=\left(\mathrm{U}_{1} \mathrm{~V}_{1}\right)\left(\mathrm{U}_{2} \mathrm{~V}_{2}\right) \ldots \ldots \ldots\left(\mathrm{U}_{\mathrm{n}} \mathrm{V}_{\mathrm{n}}\right)
$$

The final step to compute the forward kinematics is calculate the transformation ${ }_{n}^{0} T$ by the following formulation [3]

$$
{ }_{\mathrm{n}}^{0} \mathrm{~T}={ }_{1}^{0} \mathrm{~T} \cdot{ }_{2}^{1} \mathrm{~T} \cdot{ }_{3}^{2} \mathrm{~T} \ldots \ldots \cdot{ }_{\mathrm{n}}^{\mathrm{n}-1} \mathrm{~T}=\left[\begin{array}{cc}
\mathrm{R}_{\mathrm{n}}^{0} & 0 \\
0 & 1
\end{array}\right]
$$

SLIDING MODE CONTROLLER: A significant challenge in control algorithms is a linear behavior controller design for nonlinear systems. When system works with various parameters and hard nonlinearities this technique is very useful in order to be implemented easily but it has some limitations such as working near the system operating point[12]. Some of nonlinear systems which work in industrial processes are controlled by linear PID controllers, but the design of linear controller for spherical motors are extremely difficult because they are nonlinear, uncertain and MIMO[33-55]. To reduce above challenges the nonlinear robust controllers is used to systems control. Sliding mode controller (SMC) is one of the powerful nonlinear robust controllers, although this controller has been analyzed by many researchers but the first proposed was in the 1950 [12-33].This controller is used in wide range areas such as in robotics, in control process, in aerospace applications and in power converters because it has an acceptable control performance and solve some main challenging topics in control such as resistivity to the external disturbance. The lyapunov formulation can be written as follows,

$$
\mathrm{V}=\frac{1}{2} \mathrm{~S}^{\mathrm{T}} \cdot \mathrm{H} \cdot \mathrm{S}
$$

The derivation of $V$ can be determined as,

$$
\dot{\mathrm{V}}=\frac{1}{2} \mathrm{~S}^{\mathrm{T}} \cdot \dot{\mathrm{H}} \cdot \mathrm{S}+\mathrm{S}^{\mathrm{T}} \mathrm{H} \dot{\mathrm{S}}
$$

The dynamic equation of spherical motor can be written based on the sliding surface as

$$
H \dot{S}=-V S+H \dot{S}+V S-\tau
$$

It is assumed that

$$
S^{T}(\dot{H}-2 V) S=0
$$

by substituting (11) in (10)

$$
\begin{aligned}
& \dot{V}=\frac{1}{2} S^{T} \dot{H} S-S^{T} V S+S^{T}(H \dot{S}+V S- \\
& \tau)=S^{T}(H \dot{S}+V S-\tau)
\end{aligned}
$$

Suppose the control input is written as follows

$$
\begin{aligned}
\hat{\tau}=\widehat{\tau_{e q}}+\widehat{\tau_{d l s}}= & {\left[\widehat{H^{-1}}(\widehat{V})+\dot{S}\right] \widehat{H} } \\
& +K \cdot \operatorname{sgn}(S)+K_{v} S
\end{aligned}
$$

By replacing the equation (14) in (13)

$$
\begin{aligned}
& \dot{V}=S^{T}\left(H \dot{S}+V S-\widehat{H} \dot{S}-\hat{V} S-K_{v} S-\right. \\
& K \operatorname{sgn}(S)=S^{T}\left(H \dot{S}+\tilde{V} S-K_{v} S-\right. \\
& K \operatorname{Sggn}(S))
\end{aligned}
$$

It is obvious that

$$
\left|\widetilde{H} \dot{S}+\tilde{V} S-K_{v} S\right| \leq|\widetilde{H} \dot{S}|+|\tilde{V} S|+\left|K_{v} S\right|
$$

The Lemma equation in spherical motor system can be written as follows

$$
\begin{aligned}
& K_{u}=\left[|\widetilde{H} \dot{S}|+|V S|+\left|K_{v} S\right|+\eta\right]_{i} \\
& , i=1,2,3,4, \ldots
\end{aligned}
$$

The equation (12) can be written as

$$
K_{u} \geq\left|\left[H \dot{S}+V S-K_{v} S\right]_{i}\right|+\eta_{i}
$$

Therefore, it can be shown that

$$
\dot{V} \leq-\sum_{i=1}^{n} \eta_{i}\left|S_{i}\right|
$$

Based on above discussion, the control law for spherical motor is written as: 


$$
U=U_{e q}+U_{\text {swith }}
$$

Where, the model-based component $\boldsymbol{U}_{\boldsymbol{e}}$ is the nominal dynamics of systems and $\boldsymbol{U}_{\boldsymbol{e q}}$ can be calculate as follows:

$$
U_{e q}=\left[H^{-1}(B+C)+\dot{S}\right] H
$$

$\boldsymbol{U}_{\text {SWITCH}}$ is computed as;

$$
U_{\text {switch }}=K \cdot \operatorname{SGN}(\lambda e+\dot{e})
$$

by replace the formulation (22) in (20) the control output can be written as;

$$
U=U_{e q}+K \cdot \operatorname{SGN}(S)
$$

By (23) and (21) the sliding mode control of spherical motor is calculated as;

$$
U=\left[H^{-1}(B+C)+\dot{S}\right] H+K \cdot \operatorname{SGN}(S)
$$

Figure 3 shows the conventional sliding mode controller for three dimension of spherical motor.

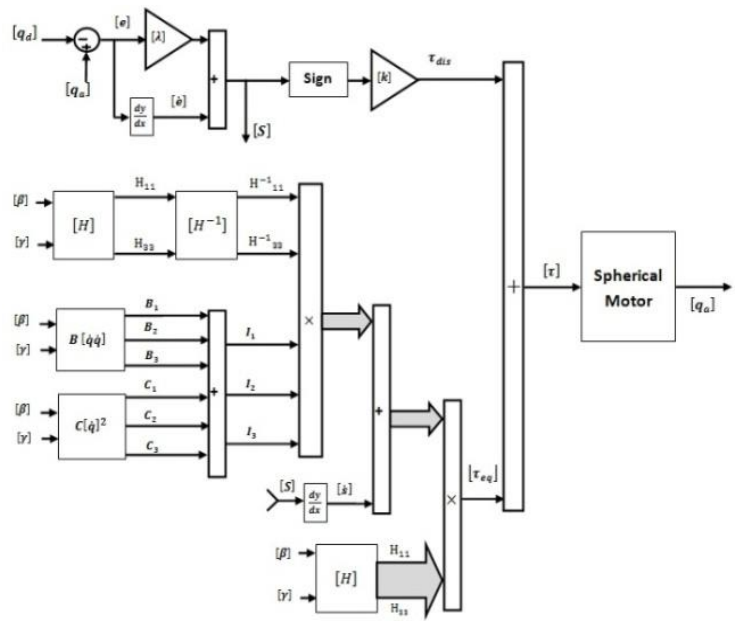

Fig 3: Sliding Mode Control: 3 DOF spherical motor

FUZZY LOGIC THEORY: This section provides a review about foundation of fuzzy logic based on [56- 66]. Supposed that $U$ is the universe of discourse and $x$ is the element of $U$, therefore, a crisp set can be defined as a set which consists of different elements $(x)$ will all or no membership in a set. A fuzzy set is a set that each element has a membership grade, therefore it can be written by the following definition;

$$
A=\left\{x, \mu_{A}(x) \mid x \in X\right\} ; A \in U
$$

Where an element of universe of discourse is $x, \mu_{A}$ is the membership function (MF) of fuzzy set. The membership function $\left(\mu_{A}(x)\right)$ of fuzzy set $A$ must have a value between zero and one. If the membership function $\mu_{A}(x)$ value equal to zero or one, this set change to a crisp set but if it has a value between zero and one, it is a fuzzy set. Defining membership function for fuzzy sets has divided into two main groups; namely; numerical and functional method, which in numerical method each number has different degrees of membership function and functional method used standard functions in fuzzy sets. The membership function which is often used in practical applications includes triangular form, trapezoidal form, bell-shaped form, and Gaussian form.

Linguistic variable can open a wide area to use of fuzzy logic theory in many applications (e.g., control and system identification). In a natural artificial language all numbers replaced by words or sentences.

If - then Rule statements are used to formulate the condition statements in fuzzy logic. A single fuzzy If - then rule can be written by

$$
\text { If } x \text { is } A \text { Then } y \text { is } B
$$

where $A$ and $B$ are the Linguistic values that can be defined by fuzzy set, the If - part of the part of " $x$ is $A$ " is called the antecedent part and the thenpart of the part of " $y$ is $B$ " is called the Consequent or Conclusion part. The antecedent of a fuzzy if-then rule can have multiple parts, which the following rules shows the multiple antecedent rules:

$$
\text { if } e \text { is } N B \text { and } \dot{e} \text { is } M L \text { then } T \text { is } L L
$$

where $e$ is error, $\dot{e}$ is change of error, $N B$ is Negative Big, $M L$ is Medium Left, $T$ is torque and $L L$ is Large Left. If - then rules have three parts, namely, fuzzify inputs, apply fuzzy operator and apply implication method which in fuzzify inputs the fuzzy statements in the antecedent replaced by the degree of membership, apply fuzzy operator used when the antecedent has multiple parts and replaced by single number between 0 to 1 , this part is a degree of support for the fuzzy rule, and apply implication method used in consequent of fuzzy rule to replaced by the degree of membership. The fuzzy inference engine offers a mechanism for transferring the rule base in fuzzy set which it is divided into two most important methods, namely, Mamdani method and Sugeno method. Mamdani method is one of the common fuzzy inference systems and he designed one of the first fuzzy controllers to control of system engine. Mamdani's fuzzy inference system is divided into four major steps: fuzzification, rule evaluation, aggregation of the rule outputs and defuzzification. Michio Sugeno use a singleton as a membership function of the rule consequent part. The following definition shows the Mamdani and Sugeno fuzzy rule base

$$
\begin{array}{ccc}
\text { Mamdani } & F . R^{1}: \text { if } & x \text { is } A \text { and } \\
y & \text { is } B & \text { then } \\
\text { Sugeno } C & \text { is } C \\
& F R^{1}: \text { if } & x \text { is } A \text { and } \\
y \text { is } B \text { then } & f(x, y) \text { is } C
\end{array}
$$

When $x$ and $y$ have crisp values fuzzification calculates the membership degrees for antecedent part. 
Rule evaluation focuses on fuzzy operation $(A N D / O R)$ in the antecedent of the fuzzy rules. The aggregation is used to calculate the output fuzzy set and several methodologies can be used in fuzzy logic controller aggregation, namely, Max-Min aggregation, Sum-Min aggregation, Max-bounded product, Max-drastic product, Max-bounded sum, Max-algebraic sum and Min-max. Two most common methods that used in fuzzy logic controllers are Max-min aggregation and Sum-min aggregation. Max-min aggregation defined as below

$$
\begin{aligned}
& \mu_{U}\left(x_{k}, y_{k}, U\right)=\mu_{\cup_{i=1}^{r} F R^{i}}\left(x_{k}, y_{k}, U\right) \\
& =\max \left\{\min _{i=1}^{r}\left[\mu_{R p q}\left(x_{k}, y_{k}\right), \mu_{p_{m}}(U)\right]\right\}
\end{aligned}
$$

The Sum-min aggregation defined as below

$$
\begin{aligned}
& \mu_{U}\left(x_{k}, y_{k}, U\right)=\mu_{\cup_{i=1}^{r} F R^{i}}\left(x_{k}, y_{k}, U\right) \\
& =\sum \min _{i=1}^{r}\left[\mu_{R p q}\left(x_{k}, y_{k}\right), \mu_{p_{m}}(U)\right]
\end{aligned}
$$

where $r$ is the number of fuzzy rules activated by $x_{k}$ and $y_{k}$ and also $\mu_{\cup_{i=1}^{r} F R^{i}}\left(x_{k}, y_{k}, U\right)$ is a fuzzy interpretation of $i-t h$ rule. Defuzzification is the last step in the fuzzy inference system which it is used to transform fuzzy set to crisp set. Consequently defuzzification's input is the aggregate output and the defuzzification's output is a crisp number. Centre of gravity method (COG) and Centre of area method (COA) are two most common defuzzification methods, which $C O G$ method used the following equation to calculate the defuzzification

$$
\operatorname{COG}\left(x_{k}, y_{k}\right)=\frac{\sum_{i} U_{i} \sum_{j=1}^{r} \cdot \mu_{u}\left(x_{k}, y_{k}, U_{i}\right)}{\sum_{i} \sum_{j=1}^{r} \cdot \mu_{u}\left(x_{k}, y_{k}, U_{i}\right)}
$$

and $C O A$ method used the following equation to calculate the defuzzification

$$
\operatorname{COA}\left(x_{k}, y_{k}\right)=\frac{\sum_{i} U_{i} \cdot \mu_{u}\left(x_{k}, y_{k}, U_{i}\right)}{\sum_{i} \mu_{U} \cdot\left(x_{k}, y_{k}, U_{i}\right)}
$$

Where $\operatorname{COG}\left(x_{k}, y_{k}\right)$ and $\operatorname{COA}\left(x_{k}, y_{k}\right)$ illustrates the crisp value of defuzzification output, $U_{i} \in U$ is discrete element of an output of the fuzzy set, $\mu_{U} \cdot\left(x_{k}, y_{k}, U_{i}\right)$ is the fuzzy set membership function, and $r$ is the number of fuzzy rules.

Based on foundation of fuzzy logic methodology; fuzzy logic controller has played important rule to design nonlinear controller for nonlinear and uncertain systems [53-66]. However the application area for fuzzy control is really wide, the basic form for all command types of controllers consists of;

- Input fuzzification

(binary-to-

fuzzy[B/F $]$ conversion)

- Fuzzy rule base (knowledge base)

- Inference engine
- Output defuzzification binary[F/B]conversion).

Figure 4 shows fuzzy controller operation.

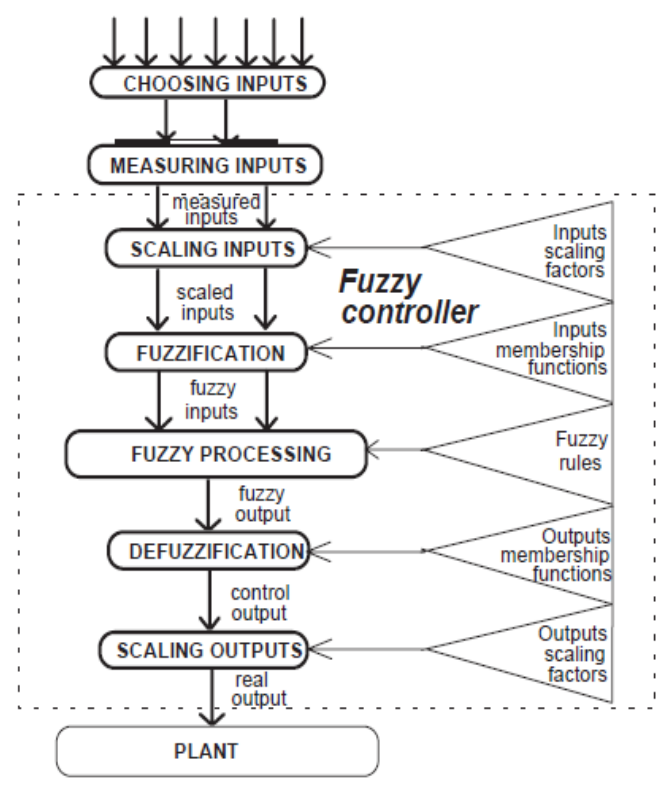

Fig 4: Fuzzy Controller operation

\section{MethodOLOGY}

The methodology in this research has two main parts:

1. Design minimum rule base PID like fuzzy logic controller.

2. Design chattering free sliding mode hybrid guidance controller.

Design PID like fuzzy controller: the first type of controller is PID like fuzzy logic controller based on 49 rule bases. However defined and number of rule base play important role to design high quality controller but system has limitation to the number of rule base to implementation and the speed of response. Based on literature PID controller can reduce or eliminate the steady state error and design stable controller. But this type of controller has three types of inputs; proportional part, integral part and derivative part. To design PID like fuzzy controller and if any input is described with seven linguistic values, and any rule has three conditions we will need 343 rules. It is too much work to write 343 rules, the speed of system is too low and design embedded controller based on FPGA or CPLD is very difficult. In PD like fuzzy controller error and change of error are the inputs and if any input is described with seven linguistic values, and any rule has two conditions we will need 49 rules. Table 1 shows the rule table of PD like fuzzy controller based on seven linguistic variables for each input and totally 49 rules. 
Table 1: Rule Table of PD like Fuzzy Controller

\begin{tabular}{|c|c|c|c|c|c|c|c|}
\hline$e^{\Delta e}$ & PB & PM & PS & $\mathbf{Z}$ & NS & NM & NB \\
\hline PB & NB & NB & NB & NB & NM & NS & $\mathbf{Z}$ \\
\hline PM & NB & NB & NB & NM & NS & $\mathbf{Z}$ & PS \\
\hline PS & NB & NB & NM & NS & $\mathbf{Z}$ & PS & PM \\
\hline $\mathbf{Z}$ & NB & NM & NS & $\mathbf{Z}$ & PS & PM & PB \\
\hline NS & NM & NS & $\mathbf{Z}$ & PS & PM & PB & PB \\
\hline NM & NS & $\mathbf{Z}$ & PS & PM & PB & PB & PB \\
\hline NB & $\mathbf{Z}$ & PS & PM & PB & PB & PB & PB \\
\hline
\end{tabular}

This table includes 49 rules. We are taking into account now not just the error but the change-of-error as well. It allows describing the dynamics of the controller. To explain how this rules set works and how to choose the rules, let us divide the set of all rules into the following five groups:

Group 1: In this group of rules both e and $\Delta \mathrm{e}$ are (positive or negative) small or zero. This means that the current value of the process output variable has deviated from the desired level (the set-point) but is still close to it. Because of this closeness the control signal should be zero or small in magnitude and is intended to correct small deviations from the set-point. Therefore, the rules in this group are related to the steady-state behavior of the process. The change-of-error, when it is Negative Small or Positive Small, shifts the output to negative or positive region, because in this case, for example, when $\mathrm{e}(\mathrm{t})$ and $\Delta \mathrm{e}(\mathrm{t})$ are both Negative Small the error is already negative and, due to the negative change-of-error, tends to become more negative. To prevent this trend, one needs to increase the magnitude of the control output.

Group 2: For this group of rules e $(\mathrm{t})$ is Positive Big or Medium which implies that actual input is significantly above the set point. At the same time since $\Delta \mathrm{e}(\mathrm{t})$ is negative, this means that actual input is moving towards the set-point. The control signal is intended to either speed up or slow down the approach to the set-point. For example, if actual input is much below the set-point $(\boldsymbol{e}(\boldsymbol{t})$ is Positive Big) and it is moving towards the set-point with a small step $(\Delta e(t)$ is Negative Small) then the magnitude of this step has to be significantly increased ( $\boldsymbol{U}$ is Negative Medium). However, when actual input is still much below the set-point $(e(t)$ is Positive Big) but it is moving towards the set-point very fast $(\Delta e(t)$ is Negative Big) no control action can be recommended because the error will be compensated due to the current trend.

Group 3: For this group of rules actual output is either close to the set-point $(e(t)$ is Positive Small, Zero, Negative Small) or significantly above it (Negative Medium, Negative Big). At the same time, since $\Delta e(t)$ is negative, actual input is moving away from the set-point. The control here is intended to reverse this trend and make actual input, instead of moving away from the setpoint, start moving towards it. So here the main reason for the control action choice is not just the current error but the trend in its change.
Group 4: For this group of rules $e(t)$ is Negative Medium or Big, which means that actual input is significantly below the set-point. At the same time, since $\Delta e(t)$ is positive, actual input is moving towards the set-point. The control is intended to either speed up or slow down the approach to the set-point. For example, if actual input is much above the set-point $(e(t)$ is Negative $\mathrm{Big})$ and it is moving towards the set-point with a somewhat large step $(\Delta e(t)$ is Positive Medium), then the magnitude of this step has to be only slightly enlarged (output is Negative Small).

Group 5: The situation here is similar to the Group 3 in some sense. For this group of rules $e(t)$ is either close to the set-point (Positive Small, Zero, Negative Small) or significantly above it (Positive Medium, Positive Big). At the same time since $\Delta e(t)$ is positive actual input is moving away from the set-point. This control signal is intended to reverse this trend and make actual input instead of moving away from the set-point start moving towards it. In PI like controller error and integral of error are the inputs. Based on above discussion the PID-like fuzzy controller can be constructed as a parallel structure of a PD-like fuzzy controller and a PI-like controller with the output approximated as:

$$
U_{P I D}=\left(\frac{K_{p_{a}}}{2} e+K_{V_{a}} \dot{e}\right)+\left(\frac{K_{p_{a}}}{2} e+K_{I} \sum e\right)
$$

In this type of design, we have 49 rule bases for PD like fuzzy controller. After design the PID like fuzzy controller the second objective is commenced.

Design chattering free sliding mode controller: To reduce/eliminate the chattering phenomenon parallel baseline methodology is applied to switching part of sliding mode controller. Baseline control is a nonlinear second order controller and this controller is worked based on linear type of controller. Baseline controller has two main parts:

- The first type controller which most of time is PID controller.

- The second controller that is PD or PI type controller.

Figure 5 shows the baseline controller to reduce/eliminate the chattering. The formulation of baseline controller is;

$$
\begin{aligned}
U_{\text {Baseline }}=\left(K_{p} e\right. & \left.+K_{V} \dot{e}+K_{I} \sum e\right) \\
& \times\left(K_{p} e+K_{V} \dot{e}\right)
\end{aligned}
$$

The formulation of modified chattering free sliding mode controller is;

$$
\begin{aligned}
U_{\text {Modify-SMC }}=[ & \left(K_{p} e+K_{V} \dot{e}+K_{I} \sum e\right) \\
& \times\left(K_{p} e+K_{V} \dot{e}\right)+K \\
& \cdot \operatorname{SGN}(S)] \\
& +\left[H^{-1}(B+C)+\dot{S}\right] H
\end{aligned}
$$




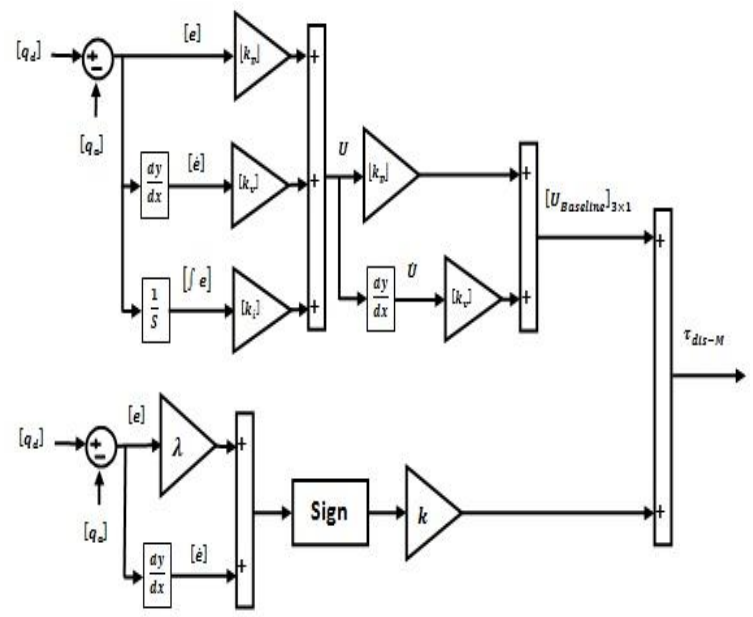

Fig 5: Block diagram of modified baseline sliding mode controller

However this controller work in many applications but it cannot guarantee reliability and stability. To solve this challenge nonlinear chattering free sliding mode controller is used to improve the reliability and stability. Figure 6 Shows the PID like fuzzy hybrid sliding mode guidance controller for spherical motor.

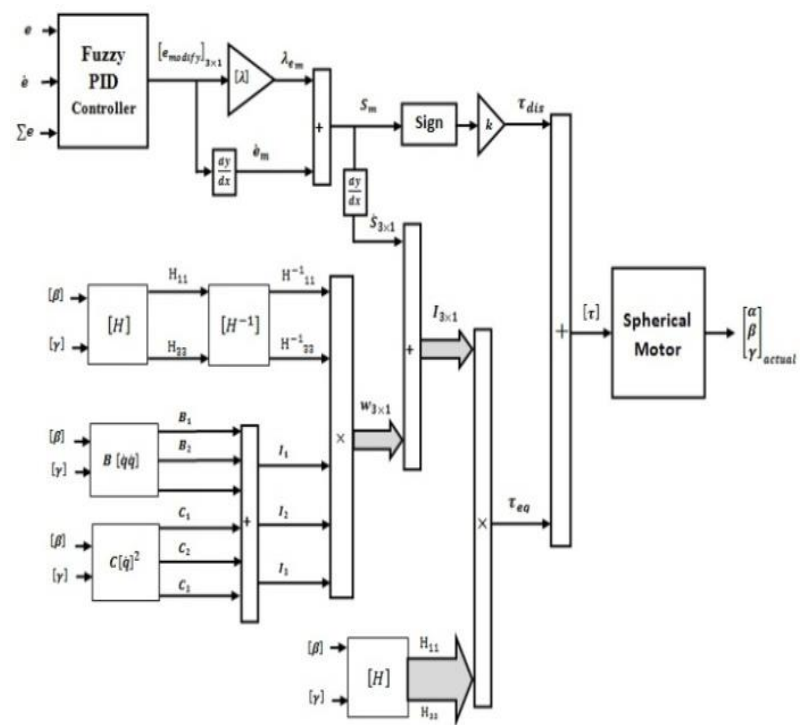

Fig 6: Block Diagram of Fuzzy Hybrid Modify chattering free Sliding Mode Guidance Controller

Based on fuzzy logic methodology

$$
f(x)=U_{\text {fuzzy }}=\sum_{l=1}^{M} \theta^{T} \zeta(x)
$$

where $\theta^{T}$ is adjustable parameter (gain updating factor) and $\zeta(x)$ is defined by;

$$
\zeta(x)=\frac{\sum_{i} \mu\left(x_{i}\right) x_{i}}{\sum_{i} \mu\left(x_{i}\right)}
$$

Where $\mu\left(x_{i}\right)$ is membership function. According to the hybrid control theory;

$$
\begin{aligned}
\tau=U_{\text {fuzzy }}+U_{\text {Modify }-S M C} & \\
& =\sum_{l=1}^{M} \theta^{T} \zeta(x) \\
& +\left[\left(K_{p} e+K_{V} \dot{e}\right.\right. \\
& \left.+K_{I} \sum e\right) \\
& \times\left(K_{p} e+K_{V} \dot{e}\right)+K \\
& \cdot \operatorname{SGN}(S)] \\
& +\left[H^{-1}(B+C)\right. \\
& +\dot{S}] H
\end{aligned}
$$

\section{RESULTS AND DISCUSSION}

PID like fuzzy hybrid sliding mode guidance controller was tested to Step response trajectory. This simulation is used to control position of spherical motor without and with external disturbance. The simulation was implemented in MATLAB/SIMULINK environment. These systems are tested by band limited white noise with a predefined $40 \%$ of relative to the input signal amplitude. This type of noise is used to external disturbance in continuous and hybrid systems and applied to nonlinear dynamic of these controllers.

Tracking performances: stability, reliability and robust are three main important experiments under condition of certain and uncertain parameters. To test the stability, trajectory following has play important responsibility. This part focuses on comparison between conventional sliding mode control and proposed controller. Based on Figure 7, these two control method have the same performance and both of controllers have stability and reliability. This graph illustrates; conventional sliding mode controller has acceptable performance in certain system.
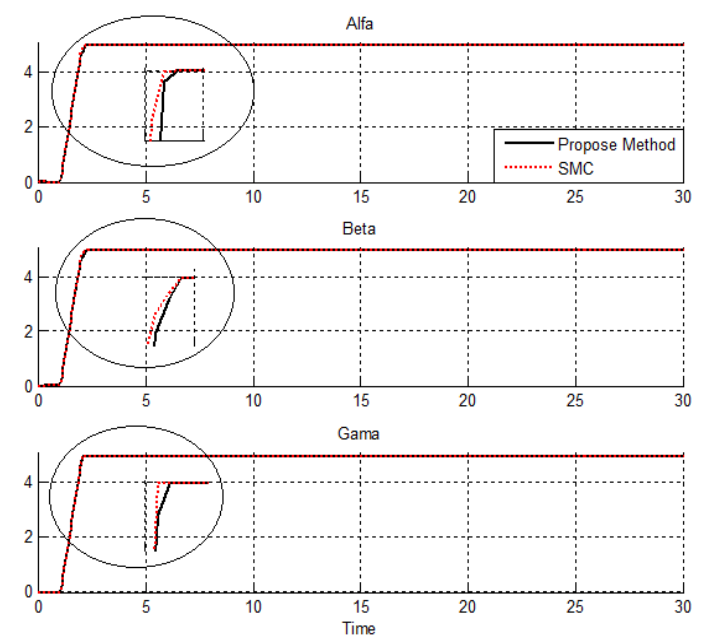

Fig 7: SMC controller and proposed method

According to Figure 7, the rise time of conventional sliding mode controller is less than proposed method. Therefore in certain condition chattering free sliding mode methodology works better than proposed method. 
Disturbance rejection: in this research $40 \%$ external disturbance applied to these two controllers. This test is used to robust checking of controllers. Figure 8 shows the power disturbance elimination in proposed method and pure SMC controller in presence of $40 \%$ external disturbance and uncertainty in parameters. According to the following graph, pure SMC has moderate fluctuations in presence of external disturbance and uncertainty because, this type of controller is has limitation in presence of uncertainty and external disturbance. The overshoot of SMC is about $15 \%$ and this controller also has moderate oscillation. It means that, sliding mode controller has challenge in uncertain condition like the other conventional controllers.
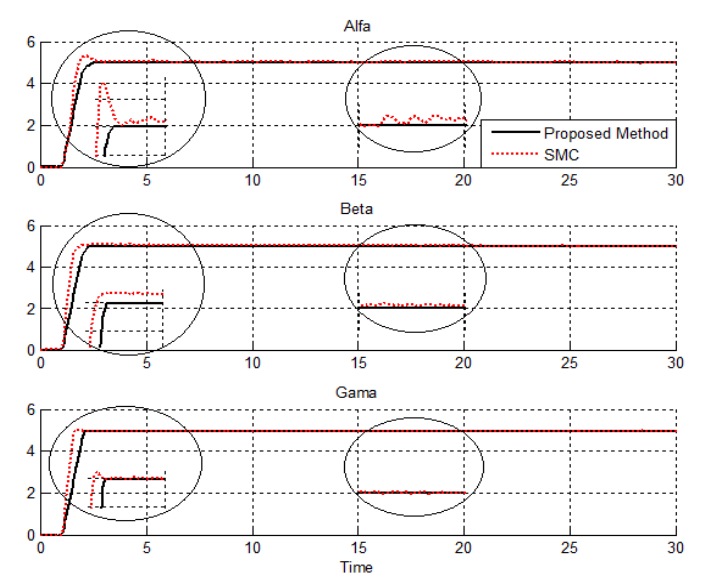

Fig 8: SMC controller and proposed method in presence of external disturbance

Proposed method can increase the controller's forceful based on guidance technique and fuzzy intelligence method.

\section{CONCLUSION}

The central issues and challenges of non linear control and estimation problems are stability, robustness and reliability. Opt for design sliding mode controller to have above objectives is a good situation in certain and partly uncertain condition. In un-limited uncertain condition, conventional sliding mode controller cannot guarantee stability and good robustness. To solve SMC challenge fuzzy logic controller is introduced based on 49 rule base. Pure fuzzy logic controller has challenge to stability and reliability. Design fuzzy hybrid sliding mode guidance controller has the below advantages:

- It is a stable, robust and reliable chattering free controller.

- The performance of proposed method in unlimited uncertainty is very good.

- This type of design can eliminate the chattering extremely well.

According to result and discussion, proposed method can eliminate the overshoot and oscillation.

\section{ACKNOWLEDGMENT}

The authors would like to thank the anonymous reviewers for their careful reading of this paper and for their helpful comments. This work was supported by the SSP Institute of Advance Science and Technology Program of Iran under grant no. 2013-Persian Gulf-2A.

\section{REFERENCES:}

[1] Vachtsevanos, G. I., Davey, K. and Lee, K. M., "Development of a Novel Intelligent Robotic Manipulator," IEEE Control System Magazine, 1987, pp.915.

[2] Davey, K., Vachtsevanos, G. I., and Powers, R., "An analysis of Fields and Torques in Spherical Induction Motors," 1EE Transactions on Magnetics, Vol. MAG-23, 1987, pp. 273-282.

[3] Foggia, A., Oliver, E., Chappuis, F., "New Three Degrees of Freedom Electromagnetic Actuator," Conference Record -lAS Annual Meeting, Vol. 35, New York, 1988.

[4] Lee, K. M., Vachtsevanos, G. and Kwan, C-K., "Development of a Spherical Wrist Stepper Motor," Proceedings of the 1988 IEEE Intemational Conference on Robotics and Automation, Philadelphia, PA. April 26-29.

[5] Lee, K. M., Pei. I., "Kinematic Analysis of a Three Degree-of-Freedom Spherical Wrist Actuator," The Fifth International Conference on Advanced Robotics, Italy, 1991.

[6] Wang, I., Jewel, G., Howe, D., "Modeling of a Novel Spherical Pennanent Magnet Actuator," Proceedings of IEEE International Conference on Robotics and Automation, Albuquerque, New Mexico, pp 1190-1195, 1997.

[7] Wang, I., Jewel, G., Howe, D., "Analysis, Design and Control of a Novel Spherical Pennanent Magnet Actuator," lEE Proceedings on Electrical Power Applications., vol. 154, no. 1, 1998.

[8] Chirikjian, G. S., and Stein, D., "Kinematic Design and Commutation of a Spherical Stepper Motor," IEEEIASME Transactions on Mechatronics, vol. 4, n 4, Piscataway, New Jersey, pp. 342-353, Dec. 1999.

[9] Kahlen, K., and De Doncker, R. W., "CW'l'ent Regulators for Multi-phase Pennanent Magnet Spherical Machines." Industry Applications Conference Record of the 2000 IEEE, vol. 3, 2000, pp. 2011-2016.

[10] Lee, K. M., Pei, I., and Gilboa, U., "On the Development of a Spherical Wrist Actuator," Proceedings of the 16th NSF Conference on Manufacturing Systems Research, Tempe AZ, January 8-12, 1990.

[11] Yang, C., Back, Y. S., "Design and Control of the 3dcgn:es of freedom actuator by Controlling the Electromagnetic Force," IEEE Transactions on Magnetics, May, 1999, pp. 3607-3609.

[12] Samira Soltani \& Farzin Piltan, "Design Artificial Nonlinear Controller Based on Computed Torque like Controller with Tunable Gain", World Applied Science Journal (WASJ), 14 (9): 1306-1312, 2011.

[13] Farzin Piltan, Mohammadali Dialame, Abbas Zare \& Ali Badri,"Design Novel Lookup Table Changed Auto Tuning FSMC:Applied to Robot Manipulator", International Journal of Engineering, 6 (1):25-41, 2012.

[14] Farzin Piltan, Mohammad Keshavarz, Ali Badri \& Arash Zargari,"Design Novel Nonlinear Controller Applied to RobotManipulator: Design New Feedback Linearization Fuzzy Controller with Minimum Rule Base Tuning 
Method", International Journal of Robotics and Automation,3 (1):1-12, 2012.

[15] Farzin Piltan, Iman Nazari, Sobhan Siamak, Payman Ferdosali,"Methodology of FPGA-Based Mathematical error-Based Tuning Sliding Mode Controller", International Journal of Control and Automation, 5(1), 89118, 2012.

[16] Farzin Piltan, Bamdad Boroomand, Arman Jahed \& Hossein Rezaie, "Methodology of Mathematical ErrorBased Tuning Sliding Mode Controller", International Journal of Engineering, 6 (2):96-117, 2012.

[17] Farzin Piltan, Sara Emamzadeh, Zahra Hivand, Fatemeh Shahriyari \& Mina Mirazaei, "PUMA-560 Robot Manipulator Position Sliding Mode Control Methods Using MATLAB/SIMULINK and Their Integration into Graduate/Undergraduate Nonlinear Control, Robotics and MATLAB Courses", International Journal of Robotics and Automation, 3(3):106-150, 2012.

[18] Farzin Piltan, Ali Hosainpour, Ebrahim Mazlomian, Mohammad Shamsodini, Mohammad H. Yarmahmoudi, "Online Tuning Chattering Free Sliding Mode Fuzzy Control Design: Lyapunov Approach", International Journal of Robotics and Automation, 3(3):77105, 2012.

[19] Farzin Piltan, Mina Mirzaei, Forouzan Shahriari, Iman Nazari, Sara Emamzadeh, "Design Baseline Computed Torque Controller", International Journal of Engineering, 6(3): 129-141, 2012.

[20] Farzin Piltan, Mohammad H. Yarmahmoudi, Mohammad Shamsodini, Ebrahim Mazlomian, Ali Hosainpour, "PUMA-560 Robot Manipulator Position Computed Torque Control Methods Using MATLAB/SIMULINK and Their Integration into Graduate Nonlinear Control and MATLAB Courses", International Journal of Robotics and Automation, 3(3): 167-191, 2012.

[21] Farzin Piltan, Hossein Rezaie, Bamdad Boroomand, Arman Jahed, "Design Robust Backstepping on-line Tuning Feedback Linearization Control Applied to IC Engine", International Journal of Advance Science and Technology, 11:40-22, 2012.

[22] Farzin Piltan, Mohammad R. Rashidian, Mohammad Shamsodini and Sadeq Allahdadi, Effect of Rule Base on the Fuzzy-Based Tuning Fuzzy Sliding Mode Controller: Applied to 2nd Order Nonlinear System", International Journal of Advanced Science and Technology, 46:39-70, 2012.

[23] Farzin Piltan, Arman Jahed, Hossein Rezaie and Bamdad Boroomand, "Methodology of Robust Linear On-line High Speed Tuning for Stable Sliding Mode Controller: Applied to Nonlinear System", International Journal of Control and Automation, 5(3): 217-236, 2012.

[24] Farzin Piltan, Bamdad Boroomand, Arman Jahed and Hossein Rezaie, "Performance-Based Adaptive Gradient Descent Optimal Coefficient Fuzzy Sliding Mode Methodology", International Journal of Intelligent Systems and Applications, vol.4, no.11, pp.40-52, 2012.

[25] Farzin Piltan, Mehdi Akbari, Mojdeh Piran, Mansour Bazregar, "Design Model Free Switching Gain Scheduling Baseline Controller with Application to Automotive Engine", International Journal of Information Technology and Computer Science, vol.5, no.1, pp.65-73, 2013.DOI: 10.5815/ijitcs.2013.01.07.

[26] Farzin Piltan, Mojdeh Piran , Mansour Bazregar, Mehdi Akbari, "Design High Impact Fuzzy Baseline Variable Structure Methodology to Artificial Adjust Fuel Ratio", International Journal of Intelligent Systems and
Applications, vol.5, no.2, pp.59-70, 2013.DOI: 10.5815/ijisa.2013.02.0.

[27] Farzin Piltan, M. Bazregar, M. kamgari, M. Akbari and M. Piran, "Adjust the Fuel Ratio by High Impact Chattering Free Sliding Methodology with Application to Automotive Engine", International Journal of Hybrid Information Technology, 6(1), 2013.

[28] Farzin Piltan, S. Zare , F. ShahryarZadeh, M. Mansoorzadeh, M. kamgari, "Supervised Optimization of Fuel Ratio in IC Engine Based on Design Baseline Computed Fuel Methodology", International Journal of Information Technology and Computer Science, vol.5, no.4, pp.76-84, 2013.DOI: 10.5815/ijitcs.2013.04.09.

[29] Farzin Piltan, M. Mansoorzadeh, S. Zare, F.Shahryarzadeh, M. Akbari, "Artificial Tune of Fuel Ratio: Design a Novel SISO Fuzzy Backstepping Adaptive Variable Structure Control", International Journal of Electrical and Computer Engineering, 3(2), 2013.

[30] M. Bazregar, Farzin Piltan, A. Nabaee and M.M. Ebrahimi, "Parallel Soft Computing Control Optimization Algorithm for Uncertainty Dynamic Systems", International Journal of Advanced Science and Technology, 51, 2013.

[31] Farzin Piltan, M.H. Yarmahmoudi, M. Mirzaei, S. Emamzadeh, Z. Hivand, "Design Novel Fuzzy Robust Feedback Linearization Control with Application to Robot Manipulator", International Journal of Intelligent Systems and Applications , vol.5, no.5, pp.1-10, 2013.DOI: 10.5815/ijisa.2013.05.01.

[32] Sh. Tayebi Haghighi, S. Soltani, Farzin Piltan, M. kamgari, S. Zare, "Evaluation Performance of IC Engine: Linear Tunable Gain Computed Torque Controller Vs. Sliding Mode Controller", International Journal of Intelligent Systems and Applications, vol.5, no.6, pp.78-88, 2013.DOI 10.5815/ijisa.2013.06.10.

[33] Amin Jalali, Farzin Piltan, M. Keshtgar, M. Jalali, "Colonial Competitive Optimization Sliding Mode Controller with Application to Robot Manipulator", International Journal of Intelligent Systems and Applications, vol.5, no.7, pp.50-56, 2013. DOI: 10.5815/ijisa.2013.07.07.

[34] Salehi, Farzin Piltan, M. Mousavi, A. Khajeh, M. R. Rashidian, "Intelligent Robust Feed-forward Fuzzy Feedback Linearization Estimation of PID Control with Application to Continuum Robot", International Journal of Information Engineering and Electronic Business, vol.5, no.1, pp.1-16, 2013. DOI: 10.5815/ijieeb.2013.01.01.

[35] Farzin Piltan, M.J. Rafaati, F. Khazaeni, A. Hosainpour, S. Soltani, "A Design High Impact Lyapunov Fuzzy PD-PlusGravity Controller with Application to Rigid Manipulator", International Journal of Information Engineering and Electronic Business, vol.5, no.1, pp.17-25, 2013. DOI: 10.5815/ijieeb.2013.01.02.

[36] Amin Jalali, Farzin Piltan, A. Gavahian, M. Jalali, M. Adibi, "Model-Free Adaptive Fuzzy Sliding Mode Controller Optimized by Particle Swarm for Robot manipulator", International Journal of Information Engineering and Electronic Business, vol.5, no.1, pp.68-78, 2013. DOI: 10.5815/ijieeb.2013.01.08.

[37] Farzin Piltan, F. ShahryarZadeh ,M. Mansoorzadeh ,M. kamgari, S. Zare, "Robust Fuzzy PD Method with Parallel Computed Fuel Ratio Estimation Applied to Automotive Engine", International Journal of Intelligent Systems and Applications, vol.5, no.8, pp.83-92, 2013. DOI: 10.5815/ijisa.2013.08.10.

[38] Farzin Piltan, A. Nabaee, M.M. Ebrahimi, M. Bazregar, "Design Robust Fuzzy Sliding Mode Control Technique for Robot Manipulator Systems with Modeling 
Uncertainties", International Journal of Information Technology and Computer Science, vol.5, no.8, pp.123135, 2013. DOI: 10.5815/ijitcs.2013.08.12.

[39] Farzin Piltan, M. Mansoorzadeh, M. Akbari, S. Zare, F. ShahryarZadeh "Management of Environmental Pollution by Intelligent Control of Fuel in an Internal Combustion Engine" Global Journal of Biodiversity Science And Management, 3(1), 2013.

[40] M. M. Ebrahimit Farzin Piltan, M. Bazregar and A.R. Nabaee, "Intelligent Robust Fuzzy-Parallel Optimization Control of a Continuum Robot Manipulator", International Journal of Control and Automation, 6(3), 2013.

[41] O.R. Sadrnia, Farzin Piltan, M. Jafari, M. Eram and M. Shamsodini, "Design PID Estimator Fuzzy plus Backstepping to Control of Uncertain Continuum Robot", International Journal of Hybrid Information Technology, 6(4), 2013

[42] AminJalali, Farzin Piltan, H. Hashemzadeh, A. Hasiri, M.R Hashemzadeh, "Design Novel Soft Computing Backstepping Controller with Application to Nonlinear Dynamic Uncertain System", International Journal of Intelligent Systems and Applications, vol.5, no.10, pp.93105, 2013. DOI: 10.5815/ijisa.2013.10.12.

[43] M. Moosavi, M. Eram, A. Khajeh, O. Mahmoudi and Farzin Piltan, "Design New Artificial Intelligence Base Modified PID Hybrid Controller for Highly Nonlinear System", International Journal of Advanced Science and Technology, 57, 2013.

[44] S. Zahmatkesh, Farzin Piltan, K. Heidari, M. Shamsodini, S. Heidari, "Artificial Error Tuning Based on Design a Novel SISO Fuzzy Backstepping Adaptive Variable Structure Control" International Journal of Intelligent Systems and Applications, vol.5, no.11, pp.34-46, 2013. DOI: $10.5815 /$ ijisa.2013.11.04.

[45] S. Heidari, Farzin Piltan, M. Shamsodini, K. Heidari and S. Zahmatkesh, "Design New Nonlinear Controller with Parallel Fuzzy Inference System Compensator to Control of Continuum Robot Manipulator",International Journal of Control and Automation, 6(4), 2013.

[46] FarzinPiltan, M. Kamgari, S. Zare, F. ShahryarZadeh, M. Mansoorzadeh, "Design Novel Model Reference Artificial Intelligence Based Methodology to Optimized Fuel Ratio in IC Engine", International Journal of Information Engineering and Electronic Business, vol.5, no.2, pp.44-51, 2013. DOI: $10.5815 /$ ijieeb.2013.02.07.

[47] Farzin Piltan, Mehdi Eram, Mohammad Taghavi, Omid Reza Sadrnia, Mahdi Jafari,"Nonlinear Fuzzy Model-base Technique to Compensate Highly Nonlinear Continuum Robot Manipulator", IJISA, vol.5, no.12, pp.135-148, 2013. DOI: $10.5815 /$ ijisa.2013.12.12.

[48] Amin Jalali, Farzin Piltan, Mohammadreza Hashemzadeh, Fatemeh BibakVaravi, Hossein Hashemzadeh,"Design Parallel Linear PD Compensation by Fuzzy Sliding Compensator for Continuum Robot", IJITCS, vol.5, no.12, pp.97-112, 2013. DOI: 10.5815/ijitcs.2013.12.12.

[49] Farzin Piltan, A. Hosainpour, S. Emamzadeh, I. Nazari, M. Mirzaie, "Design Sliding Mode Controller of with Parallel Fuzzy Inference System Compensator to Control of Robot Manipulator", International Journal of Robotics and Automation, Vol. 2, No. 4, December 2013, pp. 149 162.

[50] Farzin Piltan, Mahdi Jafari, Mehdi Eram, Omid Mahmoudi, Omid Reza Sadrnia, "Design Artificial Intelligence-Based Switching PD plus Gravity for Highly Nonlinear Second Order System", International Journal of Engineering and Manufacturing, vol.3, no.1, pp.38-57, 2013.DOI: 10.5815/ijem.2013.01.04.
[51] Farzin Piltan, Sara Emamzadeh, Sara Heidari, Samaneh Zahmatkesh, Kamran Heidari, "Design Artificial Intelligent Parallel Feedback Linearization of PID Control with Application to Continuum Robot", International Journal of Engineering and Manufacturing, vol.3, no.2, pp.51-72, 2013.DOI: 10.5815/ijem.2013.02.04.

[52] Mohammad Mahdi Ebrahimi, Farzin Piltan, Mansour Bazregar, AliReza Nabaee,"Artificial Chattering Free online Modified Sliding Mode Algorithm: Applied in Continuum Robot Manipulator", International Journal of Information Engineering and Electronic Business, vol.5, no.5, pp.57-69, 2013. DOI: 10.5815/ijieeb.2013.05.08.

[53] Arman Jahed, Farzin Piltan, Hossein Rezaie, Bamdad Boroomand, "Design Computed Torque Controller with Parallel Fuzzy Inference System Compensator to Control of Robot Manipulator", International Journal of Information Engineering and Electronic Business, vol.5, no.3, pp.66-77, 2013. DOI: 10.5815/ijieeb.2013.03.08.

[54] Mohammad Shamsodini, Farzin Piltan, Mahdi Jafari, Omid reza Sadrnia, Omid Mahmoudi,"Design Modified Fuzzy Hybrid Technique: Tuning By GDO", IJMECS, vol.5, no.8, pp.58-72, 2013.DOI: 10.5815/ijmecs.2013.08.07.

[55] Mahdi Mirshekaran,Farzin Piltan,Zahra Esmaeili,Tannaz Khajeaian,Meysam Kazeminasab,"Design Sliding Mode Modified Fuzzy Linear Controller with Application to Flexible Robot Manipulator", IJMECS, vol.5, no.10, pp.53-63, 2013.DOI: 10.5815/ijmecs.2013.10.07.

[56] Meysam Kazeminasab, Farzin Piltan, Zahra Esmaeili, Mahdi Mirshekaran, Alireza Salehi ,"Design Parallel Fuzzy Partly Inverse Dynamic Method plus Gravity Control for Highly Nonlinear Continuum Robot", IJISA, vol.6, no.1, pp.112-123, 2014. DOI: 10.5815/ijisa.2014.01.12.

[57] Mansour Bazregar, Farzin Piltan, Mehdi Akbari, Mojdeh Piran," Management of Automotive Engine Based on Stable Fuzzy Technique with Parallel Sliding Mode Optimization", IJITCS, vol.6, no.1, pp.101-107, 2014. DOI: 10.5815/ijitcs.2014.01.12.

[58] Mojdeh Piran, Farzin Piltan, Mehdi Akbari, Mansour Bazregar," Quality Model and Artificial Intelligence Base Fuel Ratio Management with Applications to Automotive Engine", IJISA, vol.6, no.2, pp.76-87, 2014. DOI: 10.5815/ijisa.2014.02.10.

[59] Ali Shahcheraghi, Farzin Piltan, Masoud Mokhtar, Omid Avatefipour, Alireza Khalilian," Design a Novel SISO Offline Tuning of Modified PID Fuzzy Sliding Mode Controller", IJITCS, vol.6, no.2, pp.72-83, 2014. DOI: 10.5815/ijitcs.2014.02.10.

[60] Kamran Heidari, Farzin Piltan, Samaneh Zahmatkesh, Sara Heidari, Mahdi Jafari," Design High Efficiency Intelligent Robust Backstepping Controller ", IJIEEB, vol.5, no.6, pp.22-32, 2013. DOI: 10.5815/ijieeb.2013.06.03.

[61] Zahra Esmaieli, Farzin Piltan, Meysam Kazeminasab, Ali Reza Salehi, Mahdi Mirshekaran,"Design Intelligent Robust Back stepping Controller", IJMECS, vol.6, no.1, pp.53-64, 2014.DOI: 10.5815/ijmecs.2014.01.06.

[62] Omid Avatefipour, Farzin Piltan, Mahmoud Reza Safaei Nasrabad, Ghasem Sahamijoo, Alireza Khalilian,"Design New Robust Self Tuning Fuzzy Backstopping Methodology", IJIEEB, vol.6, no.1, pp.49-61, 2014. DOI: 10.5815/ijieeb.2014.01.06.

[63] Alireza Siahbazi, Ali Barzegar, Mahmood Vosoogh, Abdol Majid Mirshekaran, Samira Soltani,"Design Modified Sliding Mode Controller with Parallel Fuzzy Inference System Compensator to Control of Spherical Motor", IJISA, vol.6, no.3, pp.12-25, 2014. DOI: 10.5815/ijisa.2014.03.02. 
[64] Narges Gholami Mozafari, Farzin Piltan, Mohammad Shamsodini, Azita Yazdanpanah, Ali Roshanzamir,"On Line Tuning Premise and Consequence FIS Based on Lyaponuv Theory with Application to Continuum Robot", IJISA, vol.6, no.3, pp.96-110, 2014. DOI: 10.5815/ijisa.2014.03.10.

[65] Mansour Bazregar, Farzin Piltan, AliReza Nabaee, MohammadMahdi Ebrahimi,"Design Modified Fuzzy PD Gravity Controller with Application to Continuum Robot", IJITCS, vol.6, no.3, pp.82-94, 2014. DOI: 10.5815/ijitcs.2014.03.10.

[66] Arzhang Khajeh, Farzin Piltan, Mohammad Reza Rashidian, Afsaneh Salehi, Ehsan pouladi ,"Design New Intelligent PID like Fuzzy Backstepping Controller ", IJMECS, vol.6, no.2, pp.15-26, 2014.DOI: 10.5815/ijmecs.2014.02.03.

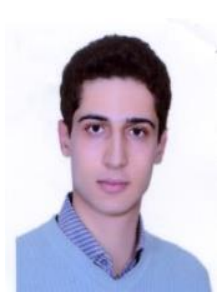

Mojtaba Yaghoot is currently working as a primary researcher in Control and Robotic $\mathrm{Lab}$ at the institute of advance science and technology, IRAN SSP research and development Center. His current research interests are in the area of nonlinear control, artificial control system and robotics, and spherical motor.

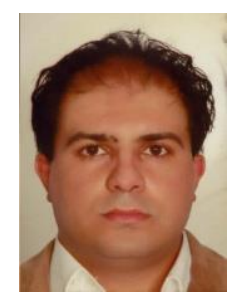

Farzin Piltan was born on 1975, Shiraz, Iran. In 2004 he is jointed Institute of Advance Science and Technology, Research and Development Center, IRAN SSP. Now he is a dean of Intelligent Control and Robotics Lab. In addition to 7 textbooks, Farzin Piltan is the main author of more than 100 scientific papers in refereed journals. He is editorial review board member for 'international journal of control and automation (IJCA), Australia, ISSN: 2005-4297; 'International Journal of Intelligent System and Applications (IJISA)', Hong Kong, ISSN: 20749058; 'IAES international journal of robotics and automation,
Malaysia, ISSN: 2089-4856; 'International Journal of Reconfigurable and Embedded Systems', Malaysia, ISSN:20894864. His current research interests are nonlinear control, artificial control system and applied to FPGA, robotics and artificial nonlinear control and IC engine modeling and control.

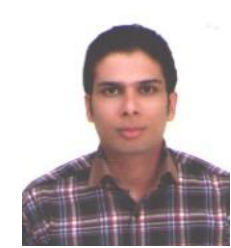

Meysam Esmaeili is currently working as a co researcher in Control and Robotic Lab at the institute of advance science and technology, IRAN SSP research and development Center. He is a Master in field of Electronic Engineering from Azad University, IRAN. His current research interests are in the area of nonlinear control, artificial control system and robotics, and spherical motor.

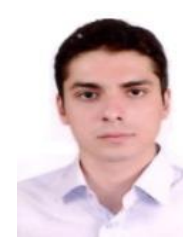

Mohammad Ali Tayebi is currently working as a co researcher in Control and Robotic Lab at the institute of advance science and technology, IRAN SSP research and development Center. He is a Master in field of Control engineering from Azad University, IRAN. His current research interests are in the area of nonlinear control, artificial control system and robotics, and spherical motor.

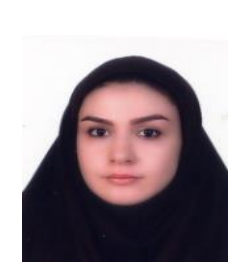

Mahsa Piltan is currently working as a co researcher in Control and Robotic Lab at the institute of advance science and technology, IRAN SSP research and development Center. She is a Master in field of communication engineering from Azad University, IRAN. Her current research interests are in the area of nonlinear control, artificial control system and robotics, and spherical motor.

How to cite this paper: Mojtaba Yaghoot, Farzin Piltan, Meysam Esmaeili, Mohammad Ali Tayebi, Mahsa Piltan,"Design Intelligent Robust Model-base Sliding Guidance Controller for Spherical Motor", IJMECS, vol.6, no.3, pp.61-72, 2014.DOI: 10.5815/ijmecs.2014.03.08 